\title{
Laparoscopic Retrieval of a Migrated Intrauterine Contraceptive Device
}

\author{
Eftekhar Hassan Al-Ojaimi ${ }^{1}$, Shafeeqa Ali Ebrahim ${ }^{2}$
}

\begin{abstract}
Introduction: Intrauterine contraceptive device (IUCD) migration consequent to perforation of the uterus is not very common, but is one of the more serious complications.

Case descriptions: We described two cases of migrated IUCD, at two distinct sites in the pelvic cavity, one was located in the pouch of Douglas embedded behind the left ovary and tube which was adherent to the posterior uterine wall and another was in the left mesovarium between the ovary and the tube. Both IUCDs were successfully removed laparoscopically without any complication.

Conclusion: Migrated IUCDs should always be removed once the diagnosis is made to prevent serious complications. Laparoscopic approach is a successful and preferred choice of treatment in selected cases.

Keywords: Intrauterine device, Laparoscopy, Migration, Uterine perforation.

World Journal of Laparoscopic Surgery (2020): 10.5005/jp-journals-10033-1409
\end{abstract}

\section{INTRODUCTION}

Intrauterine contraceptive device (IUCD) is one of the most widely used forms of contraception, predominantly in the developing countries. This method has the benefits in terms of affordability, as also being very effective, long-lasting, and reversible but it is not risk free. Insertion of an IUCD is associated with some complications like cramping abdominal pain, irregular and sometimes heavy vaginal bleeding, expulsion, and even serious complications, such as infection and pelvic inflammatory disease, retraction back to the cervix or uterine cavity, and subsequent uterine perforation. ${ }^{1,2}$ The incidence of uterine wall perforations is estimated to be around 0.2-9.6 per thousand insertions. ${ }^{2}$ A migrated IUCD can be found in different positions in the pelvic or abdominal cavity, which may cause different complications and morbidities or may be asymptomatic. Herein, we presented two cases of migrated IUCD, at two distinct sites in the pelvic cavity, who underwent successful laparoscopic procedure with IUCD removal.

\section{Case Descriptions}

\section{Case 1}

A 28-year-old para 2 with 2 living issues presented with lower abdominal pain, dull aching in character, for 4 months and stated that she was not able to feel the IUCD thread for 14 days prior to presentation. She had the IUCD inserted 2 years ago, 1 month after her second delivery during the lactational period. The IUCD thread could not be visualized on per speculum examination. On per vaginal examination, cervical motion tenderness was elicited with nodularity and tenderness felt in the pouch of Douglas. The patient underwent ultrasonography and the IUCD was located in the pelvis with minimal free fluid outside the uterine cavity. The patient was then subjected to a laparoscopic examination. Following general anesthesia, pneumoperitoneum was established and three ports were made. A subumbilical incision was made for the primary port and a $10 \mathrm{~mm}$ cannula placed for the laparoscope. Another two $5 \mathrm{~mm}$ secondary ports were put in the right and left lower quadrants for the accessory instruments. In laparoscopy, Nova-T type IUCD along
${ }^{1}$ Department of Obstetrics and Gynecology, Al Zahra Hospital Dubai, Dubai, United Arab Emirates

${ }^{2}$ Department of Obstetrics and Gynecology, Salmaniya Medical Complex, Ministry of Health, Kingdom of Bahrain

Corresponding Author: Eftekhar Hassan Al-Ojaimi, Department of Obstetrics and Gynecology, Al Zahra Hospital Dubai, Dubai, United Arab Emirates, Phone: +971566608555, e-mail: eojaimi@gmail.com

How to cite this article: Hassan Al-Ojaimi E, Ebrahim SA. Laparoscopic Retrieval of a Migrated Intrauterine Contraceptive Device. World J Lap Surg 2020;13(2):84-86.

Source of support: Nil

Conflict of interest: None

with the string was seen in the pouch of Douglas embedded behind the left ovary and tube which was adherent to the posterior wall of the uterus (Fig. 1). The adhesions were released and the IUCD was

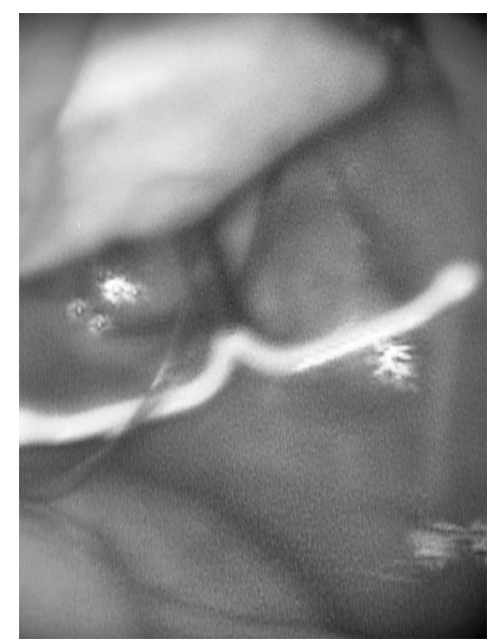

Fig. 1: Nova-T type intrauterine contraceptive device along with the string in pouch of Douglas 


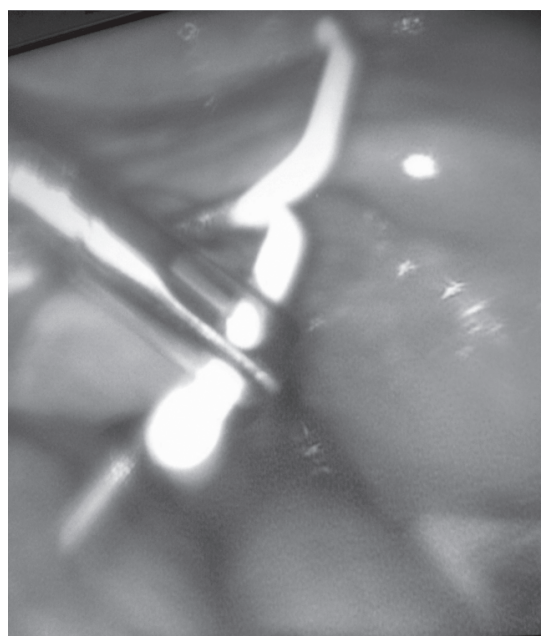

Fig. 2: Nova-T type intrauterine contraceptive device embedded in the left mesovarium between the ovary and tube

subsequently grasped and removed. The procedure was uneventful and the patient was discharged the next day.

\section{Case 2}

A 39-year-old para 5 with 4 living issues presented with severe chronic pelvic pain and deep dyspareunia for the last 2 months. She had an IUCD inserted one and a half years ago toward the end of the menstrual cycle. On per speculum examination, the IUCD thread was not seen. By ultrasonographic examination, the IUCD could not be localized inside the uterine cavity. Plain X-ray was performed and it showed the IUCD toward the left side of the pelvis outside the uterus. Laparoscopic removal of the IUCD was planned. The procedure was performed as mentioned above in Case 1. On laparoscopy, Nova-T type IUCD was seen in the pelvis embedded in the left mesovarium between the ovary and the tube (Fig. 2). The IUCD was grasped and gently removed without complication. She had uneventful recovery and was discharged home same day.

\section{Discussion}

During the insertion of IUCDs, perforation of the uterine wall is an uncommon but a very serious complication. The IUCD is usually known to perforate either the fundus, body of the uterus, or wall of the cervix. Uterine perforation can be complete or only partial. A complete perforation is when all uterine layers (endometrium, myometrium, and serosa) are perforated, as in both the cases described here. Less commonly, a partial perforation occurs, where the IUCD penetrates only the myometrial layer of the uterine wall. While the primary cause is usually idiopathic, uterine perforation can be associated with operator inexperience, IUCD, and patientrelated factors. The design and structural characteristics of the IUCD together with the nature and rigidness or malleability of the inserter are the IUCD-related factors. Patient-related factors include the parity, size of the uterus and position (acutely anteflexed or retroflexed uterus), undiagnosed pregnant uterus, timing of the insertion (early in the postpartum period, lactation, or postabortion), former uterine operations, and congenital uterine or cervical anomalies are all important determinants of potential perforation. In a case-control analysis, lactating women had $>10$-fold risk of perforation at the time of IUCD insertion than non-lactating women. ${ }^{3,4}$ Hypoestrogenic state with consequent thinning of the wall of uterus and accelerated involution of the uterus during the period of lactation could have been most likely the causes of perforation in our first patient.

Uterine perforations are reported to mainly occur in the early post-insertion period, specifically during the immediate 6 months, ${ }^{4}$ but there have been case reports of perforation seen several years after insertion. ${ }^{5,6}$ Subsequently, the IUCD can migrate into the neighboring organs or the abdominal cavity. Trauma during the insertion procedure itself, and along with the effect of chronic inflammatory reaction that causes erosion of the device through the uterine wall, can be thought to be the mechanism of IUCD migration. Delayed symptoms are presumed to be secondary migration with associated inflammatory process. Movements of the omentum may be a reason of migration of the IUCD to an adjacent organ. Migration can also be due to the growing uterus in unintended pregnancies and tubal ectopic pregnancy. The various locations where the perforated IUCDs have been found include the omentum (in 26.7\%), pouch of Douglas (in 21.5\%), lumen of the colon (in 10.4\%), uterine myometrium (in 7.4\%), broad ligament (in $6.7 \%$ ), free within the abdominal cavity (in $5.2 \%$ ), serosa of small intestine (in 4.4\%), serosa of the colon (in 3.7\%), and mesentery (in 3\%). ${ }^{7}$ The perforated IUCDs have also been found migrated to the stomach, ${ }^{1}$ colon, ${ }^{8-10}$ bladder, ${ }^{11,12}$ retroperitoneum, ${ }^{13}$ and even next to the iliac vein. ${ }^{14}$ The location of the IUCD in our second patient, embedded in the mesovarium, appears to be particularly uncommon.

Some of the patients have symptoms and/or signs suggestive of perforation such as difficulty with the insertion procedure, resulting in pain or vaginal bleeding but others may remain asymptomatic for years. Therefore, perforation should be suspected whenever the woman presents with an unintentional pregnancy or has come for removal of the IUCD and on examination, the thread cannot be seen. Ultrasonography is preferred as a first-line radiological investigation, to locate the IUCD. When an ultrasound is inconclusive, plain anteroposterior abdominal X-ray is usually performed, to confirm if the device is in the pelvis. A suspected visceral involvement may need further evaluations with computerized tomography or magnetic resonance imaging. ${ }^{15}$

Once confirmed that the IUCD is outside the uterus, the decision to leave it alone or intervene to remove the device must be made. In symptomatic patients, as in both cases presented here, all clinicians agreed that IUCD surgical removal should be performed. However, in asymptomatic patients, there still remains a controversy. Markovitch et al. advocated that, although in symptomatic patients perforated IUCD should be removed surgically, in asymptomatic patients, under certain situations, conservative management may be of benefit. ${ }^{16}$ The World Health Organization (WHO), however, has recommended that any displaced IUCDs should be removed, so as to prevent complications secondary to intraperitoneal adhesion formation or migration into surrounding organs. ${ }^{17}$ Demir et al. reported that, in cases of intra-abdominally displaced IUCD, laparoscopic removal must be the preferred choice. ${ }^{18}$ Grimaldo Arriaga et al. also encouraged immediate removal of the IUCD from the peritoneal cavity either by laparotomy or laparoscopy, along with prophylactic antimicrobials for colon preparation before elective surgery, as IUCD translocated to the peritoneal cavity may incite peritoneal or omental adhesions, uterocutaneous fistula, volvulus, and bowel perforation, which may lead to a significant morbidity. ${ }^{19}$ 
Furthermore, in deciding whether to intervene in patients who have been asymptomatic, one should consider the risks of conservative management including migration to more critical locations with subsequent need for a complicated surgery, chances of intra-abdominal abscess formation, psychological problems the patient may have, knowing about a foreign body inside her abdomen, and finally, the medicolegal consequences of a delayed management. All these supported the WHO recommendations of early surgical removal of all extrauterine IUCDs, in both symptomatic and asymptomatic patients.

Both laparotomy and laparoscopic surgeries are being performed for IUCD removal in the cases with the device migration. Laparoscopy is a preferred method as it is a minimally invasive procedure and has less complications and a shorter period of hospitalization compared to laparotomy. But laparoscopic removal is not always possible. ${ }^{7}$ In the study of Gill et al., laparoscopic removal was successful in $64.2 \%$ of all included cases of migrated IUCDs. ${ }^{7}$ The main reasons of not performing laparoscopy or converting it to laparotomy were adhesions and severe abdominal sepsis. Luckily in our both patients, we were able to safely remove the IUCDs laparoscopically without complication.

In conclusion, the possibility of perforation of the uterus should be considered in any woman who has an IUCD and the strings cannot be located, whether symptomatic or not. Surgical removal of the device, after the diagnosis is made, is recommended to prevent any subsequent serious complications. Laparoscopy is obviously preferable to laparotomy and our cases demonstrated that in selected patients, missing IUCD can be appropriately managed by laparoscopy without complication.

\section{Ethical Approval}

All procedures performed in the study involving human participants were in accordance with the ethical standards of the institutional and/or national research committee and with the 1964 Helsinki declaration and its later amendments or comparable ethical standards.

\section{Informed Consent to Participate}

Informed consent was obtained from both patients included in the study.

\section{Informed Consent to Publish}

The informed consent was obtained from both patients to publish their case.

\section{ACKNOWLedgment}

The authors are thankful to Mr Mohamed Mubarak for his excellent librarian assistance.

\section{References}

1. Bozkurt M, Yumru AE, Coskun El, et al. Laparoscopic management of a translocated intrauterine device embedded in the gastric serosa. J Pak Med Assoc 2011;61(10):1020-1022.

2. Ingec M, Kumtepe $Y$, Kadanali S, et al. A rare case of ileal embedding by an intrauterine device. Eur J Contracept Reprod Health Care 2005;10(1):29-31. DOI: 10.1080/13625180500035082.

3. Chi I. What have we learned from recent IUD studies: a researcher's perspective. Contraception 1993;48(2):81-108. DOI: 10.1016/00107824(93)90001-N.

4. Heartwell SF, Schlesselman S. Risk of uterine perforation among users of intrauterine devices. Obstet Gynecol 1983;61(1):31-36.

5. Aydogdu $\mathrm{O}$, Pulat $\mathrm{H}$. Asymptomatic far-migration of an intrauterine device into the abdominal cavity: a rare entity. CUAJ 2012;6(3): E134-E136. DOI: 10.5489/cuaj.11100.

6. Stuckey A, Dutreil P, Aspuru E, et al. Symptomatic cecal perforation by an intrauterine device with appendectomy removal. Obstet Gynecol 2005;105(5 Pt 2):1239-1241.DOI: 10.1097/01.AOG.0000157760.59342.bc.

7. Gill RS, Mok D, Hudson M, et al. Laparoscopic removal of an intra-abdominal intrauterine device: case and systematic review. Contraception 2012;85(1):15-18. DOI: 10.1016/j.contraception. 2011.04.015.

8. Gungor M, Sonmezer M, Atabekoglu C, et al. Laparoscopic management of a translocated intrauterine device perforating the bowel. J Am Assoc Gynecol Laparosc 2003;10(4):539-541. DOI: 10.1016/S1074-3804(05)60163-6.

9. Zeino MY, Wietfeldt ED, Advani V, et al. Laparoscopic removal of a copper intrauterine device from the sigmoid colon. JSLS 2011;15(4):568-570. DOI: 10.4293/108680811X13176785204661.

10. Mederos R, Humaran L, Minervini D. Surgical removal of an intrauterine device perforating the sigmoid colon: a case report. Int J Surg 2008;6(6):e60-e62. DOI: 10.1016/j.ijsu.2007.02.006.

11. Sepulveda WH, Ciuffardi I, Olivari A, et al. Sonographic diagnosis of bladder perforation by an intrauterine device. A case report. J Reprod Med 1993;38(11):911-913.

12. Dede FS, Dilbaz B, Sahin D, et al. Vesical calculus formation around a migrated copper-T 380-A. Eur J Contracept Reprod Health Care 2006;11(1):50-52. DOI: 10.1080/13625180500389349.

13. Ozgun MT, Batukan C, Serin IS, et al. Surgical management of intra-abdominal mislocated intrauterine devices. Contraception 2007;75(2):96-100. DOI: 10.1016/j.contraception.2006.09.011.

14. Roy KK, Banerjee N, Sinha A. Laparoscopic removal of translocated retroperitoneal IUD. Int J Gynaecol Obstet 2000;71(3):241-243. DOI: 10.1016/S0020-7292(00)00213-7.

15. Sun CC, Chang CC, Yu MH. Far-migrated intra-abdominal intrauterine device with abdominal pain. Taiwan J Obstet Gynecol 2008;47(2): 244-246. DOI: 10.1016/S1028-4559(08)60095-9.

16. Markovitch O, Klein Z, Gidoni Y, et al. Extrauterine mislocated IUCD: is surgical removal mandatory? Contraception 2002;66(2):105-108. DOI: 10.1016/S0010-7824(02)00327-X

17. Mechanism of action, safety and efficacy of intrauterine devices. Report of a WHO cientific group. World Health Organ Tech Rep Ser 1987;753:1-91.

18. Demir SC, Cetin MT, Ucünsak IF, et al. Removal of intra-abdominal intrauterine device by laparoscopy. Eur J Contracept Reprod Health Care 2002;7(1):20-23. DOI: 10.1080/ejc.7.1.20.23.

19. Grimaldo Arriaga J, Herrera Aviles A, Garcia Taxilaga A. Perforation of the large intestine caused by a type VII medicated copper IUCD. Ginecol Obstet Mex 1993;61:235-237. 